\title{
RHIC as a low energy collider
}

\author{
Christoph Montag ${ }^{* \dagger}$ \\ $B N L$ \\ E-mail: montag@bnl.gov
}

\begin{abstract}
The search for the critical point in the QCD phase diagram requires a center-of-mass beam energy scan in the range from roughly $5 \mathrm{GeV} /$ nucleon to $20 \mathrm{GeV} /$ nucleon. Though these energies are below its design energy, RHIC has successfully collided gold ions at center-of-mass energies of 7.7, 11.5, 14.6, and $19.6 \mathrm{GeV} /$ nucleon during phase one of the beam energy scan (BES-I). For BES-II, significantly higher luminosities are required. These will be accomplished by installation of an electron cooler. As an alternative path, installation of an internal gold target in the STAR detector has been proposed. In this presentation the RHIC performance during BES-I will be reviewed, and the design concept of the electron cooler as well as beam dynamics considerations for the design of the internal target will be presented.
\end{abstract}

9th International Workshop on Critical Point and Onset of Deconfinement

17-21 November, 2014

ZiF (Center of Interdisciplinary Research), University of Bielefeld, Germany

\footnotetext{
* Speaker.

${ }^{\dagger}$ Work supported by Brookhaven Science Associates, LLC under Contract No. DE-AC02-98CH10886 with the U.S. Department of Energy.
} 


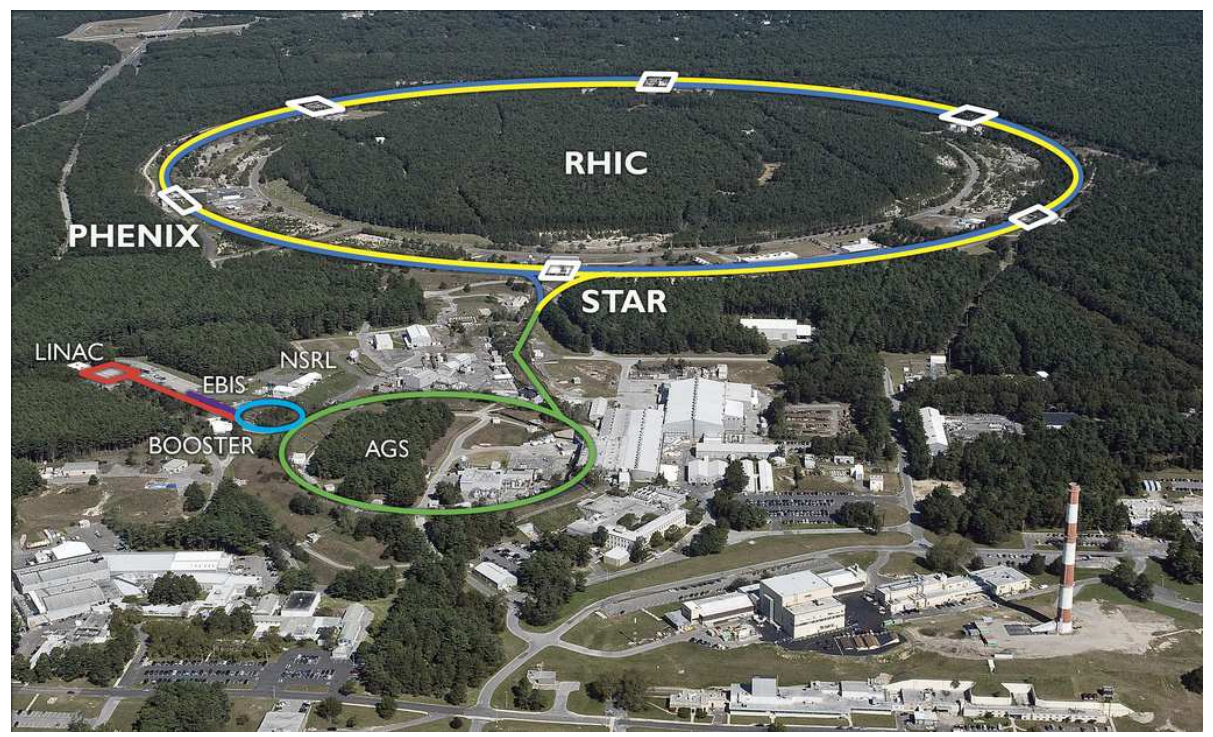

Figure 1: Aerial view of the RHIC accelerator complex with its injectors.

\section{Introduction}

The Relativistic Heavy Ion Collider (RHIC) consists of two superconducting storage rings with a circumference of $3.8 \mathrm{~km}$ that intersect in six equidistantly spaced locations around the ring circumference, as illustrated in Figure 1. Two of these interaction regions, namely the 6 o'clock and the 8 o'clock location, are equipped with the detectors STAR and PHENIX for nuclear physics research. Ion beams are generated in the electron beam ion source (EBIS) and accelerated in the Booster and AGS synchrotrons before being injected into RHIC. During nominal high energy ion operations, gold beams are injected into RHIC at an energy of $9.8 \mathrm{GeV} /$ nucleon and accelerated to a maximum energy of $100 \mathrm{GeV} /$ nucleon. Consequently, RHIC has been designed and optimized for an energy range of 9.8 to $100 \mathrm{GeV}$ /nucleon. Operating RHIC at energies below this range, as required for the QCD critical point search, is a challenging task for a variety of reasons. While the multipole errors in the superconducting accelerator magnets are minimized in the nominal energy range, they increase dramatically at energies below the nominal injection energy. Furthermore, since RHIC was not designed to operate at these low energies, magnetic measurements of those multipole errors were never performed on those magnets actually installed in the machine. All available knowledge of multipole errors below the nominal injection energy was obtained from measurements on a single spare dipole and a single spare quadrupole magnet, which have to be assumed to be good representatives of the actual magnets installed in RHIC.

While the equal ion charges in a beam repel each other, they also form parallel currents that experience an attractive force. The net effect of these two opposing forces is a repelling space charge force that scales as $1 / \gamma^{2}$. The associated defocusing effect results in a tune shift

$$
\Delta Q_{s c}=-\frac{Z^{2} r_{p}}{A} \frac{N}{4 \pi \beta \gamma^{2} \varepsilon_{N}} \frac{C}{\sqrt{2 \pi} \sigma_{s}}
$$


Table 1: Achieved beam parameters during BES-I.

\begin{tabular}{|l|r|r|r|}
\hline & $3.85 \mathrm{GeV}$ & $5.75 \mathrm{GeV}$ & $7.3 \mathrm{GeV}$ \\
\hline$\gamma$ & 4.1 & 6.1 & 7.8 \\
$I_{\text {bunch }}[1 \mathrm{e} 9]$ & 0.5 & 1.1 & 1.1 \\
$N_{\text {bunches }}$ & 111 & 111 & 111 \\
$\beta^{*}[\mathrm{~m}]$ & 6.0 & 6.0 & 3.5 \\
$\tau_{\text {beam }}[\mathrm{sec}]$ & 1000 & 1500 & 2700 \\
$\tau_{\text {lumi }}[\mathrm{sec}]$ & 400 & 1500 & 1300 \\
duration [days] & 35 & 11 & 24 \\
integrated luminosity $\left[\mu \mathrm{b}^{-1}\right]$ & 2.48 & 7.82 & 21.2 \\
\hline
\end{tabular}

which depends on the transverse amplitude of the individual particle. Here, $Z$ and $A$ are the charge state and nucleon number of the ion, $N$ the number of ions per bunch, $C$ the accelerator circumference, $\beta$ and $\gamma$ the Lorentz parameters, $\varepsilon_{N}$ the transverse beam emittance, and $\sigma_{s}$ the RMS bunch length, respectively. This space charge tune shift is largest for particles near the transverse center of the bunch, and vanishes for large amplitudes, thus resulting in a tune spread. The working point of the machine therefore has to be chosen such that the tune footprint does not overlap low-order nonlinear resonances to avoid beam loss.

During phase I of the beam energy scan (BES-I) RHIC successfully collided Au ions at three different beam energies. Parameters achieved during these runs are listed in Table 1. At low energies, the limited tuning range of the $28 \mathrm{MHz}$ RF system requires modification of the harmonic number. Due to the geometry of RHIC, with the two detectors STAR and PHENIX in the 6 o'clock and 8 o'clock positions, and the timing setup at these two detectors, only harmonic numbers that are a multiple of 9 result in collisions at both experiments, as listed in Table 2 [1]. In addition to this limitation, there is also an inaccessible gap of beam energies around the AGS transition energy, from 7.3 to $9 \mathrm{GeV}$ /nucleon. Installation of a new $9 \mathrm{MHz}$ RF system as part of the RHIC low energy cooling project will eliminate the need for modifying the harmonic number and therefore provide collisions at both experiments at all energies, while modification of the AGS transition energy is very challenging and may not be possible at all.

\section{Beam-beam effects}

When RHIC first operated at beam energies below the regular injection energy in 2010, the working point was successively lowered from the RHIC high energy ion working point of $\left(Q_{x}, Q_{y}\right)=$ $(28.23,29.22)$ to $(28.13,29.12)$ in an effort to improve the beam lifetime; further lowering of the tunes was prevented by unacceptably large amplitudes of the $10 \mathrm{~Hz}$ horizontal beam orbit jitter caused by mechanical vibrations of the low- $\beta$ quadrupole triplets [2].

During the subsequent phyics operation strong effects of the beam-beam interaction in conjunction with large space charge tune shifts of order $\Delta Q_{\mathrm{sc}}=-0.05$ were observed that manifested themselves as severe beam lifetime reductions when beams were colliding, though the beam-beam tune shift parameter was about an order of magnitude smaller than the space charge tune shift. 
Table 2: RF harmonic numbers for different beam energies, and number of simultaneous experiments.

\begin{tabular}{|c|c|c|c|}
\hline $\begin{array}{c}E_{\text {tot }} \\
{[\mathrm{GeV} / \text { nucleon }]}\end{array}$ & $\begin{array}{c}\sqrt{s_{\mathrm{NN}}} \\
{[\mathrm{GeV}]}\end{array}$ & $\begin{array}{c}\text { Harmonic } \\
\text { number }\end{array}$ & $\begin{array}{c}\text { No of } \\
\text { simultaneous } \\
\text { experiments }\end{array}$ \\
\hline $2.42-2.55$ & $4.84-5.10$ & 387 & 2 \\
$2.55-2.67$ & $5.10-5.34$ & 384 & 1 \\
$2.67-2.84$ & $5.34-5.68$ & 381 & 1 \\
$2.84-3.08$ & $5.68-6.16$ & 378 & 2 \\
$3.08-3.32$ & $6.16-6.64$ & 375 & 1 \\
$3.32-3.69$ & $6.64-7.38$ & 372 & 1 \\
$3.69-4.33$ & $7.38-8.66$ & 369 & 2 \\
$4.33-5.17$ & $8.66-10.34$ & 366 & 1 \\
$5.17-7.30$ & $10.34-14.60$ & 363 & 1 \\
7.30 & $14.60-200$ & 360 & 2 \\
$\approx 9-100$ & $14.60-200$ & 360 & 2 \\
\hline
\end{tabular}

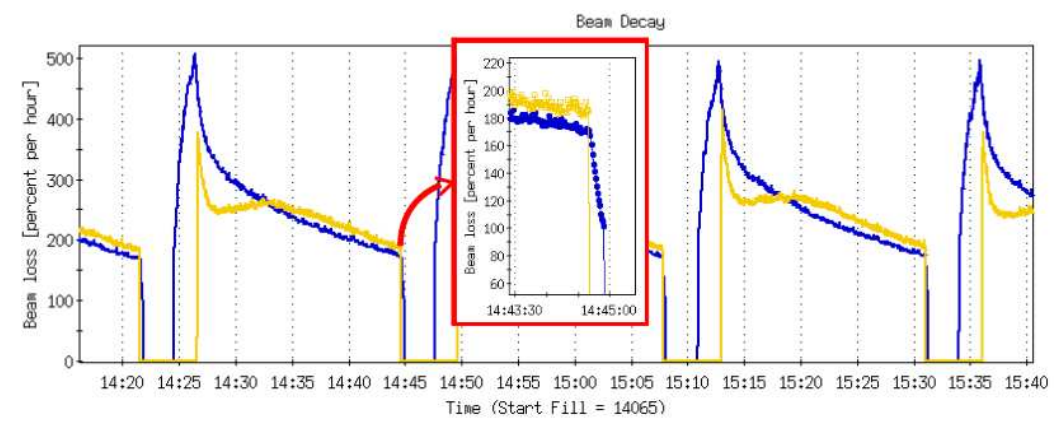

Figure 2: Beam decay rates during several $\mathrm{Au}$ beam stores at $5.75 \mathrm{GeV} /$ nucleon beam energy. The Blue beam decay rate improves dramatically as soon as the Yellow bean is dumped at the end of each store (see insert). Note that the algorithm to calculate the beam decay rate from the measured beam intensity has a time constant of $20 \mathrm{sec}$. Hence, the actual drop in instantaneous beam decay is even more dramatic than suggested by the picture.

These effects were even apparent at the end of stores when these tune shift parameters were a factor 2-3 smaller. Dumping one of the colliding beams at the end of a store resulted in an immediate and dramatic improvement in the beam lifetime of the other, as shown in Figure 2. Both experiments and simulation studies have been carried out to improve this situation by chosing a better working point. Emittance growth rates in a simple simulation model indicate that the observed beam-beam effect should be reduced at near-integer working points [3], as illustrated in Figure 3. With the advent of global fast orbit feedback at RHIC in 2011 [4] near-integer tunes have actually become accessible, and when RHIC operated at $7.3 \mathrm{GeV} /$ nucleon beam energy in FY2014 the working point was chosen at $\left(Q_{x}, Q_{y}\right)=(28.09,29.08)$.

As predicted by simulations, no effect of the beam-beam interaction on beam lifetime was 


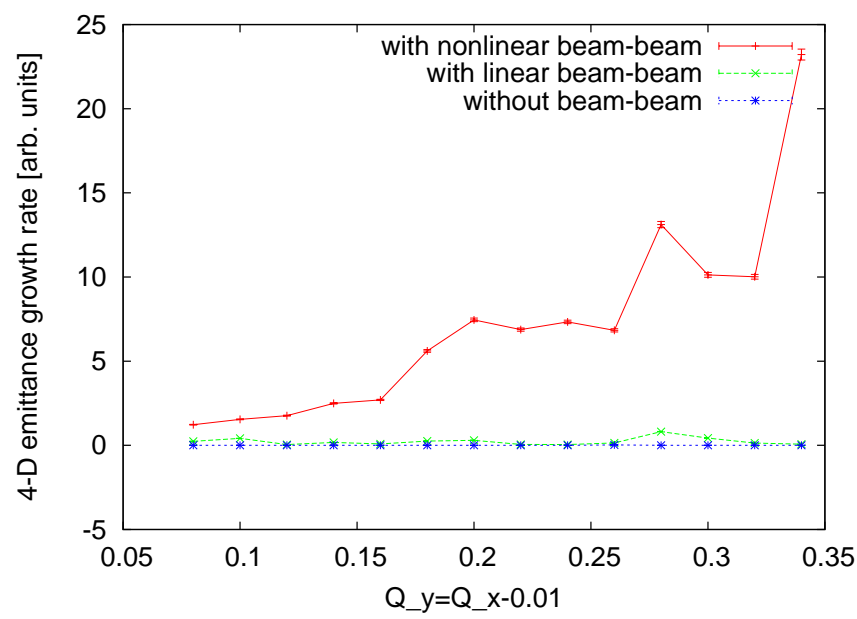

Figure 3: Simulated emittance growth rates during a tune scan with a nonlinear (red) and a linearized (green) beam-beam kick in addition to the nonlinear space charge kicks. The blue line corresponds to the case without any beam-beam interaction.

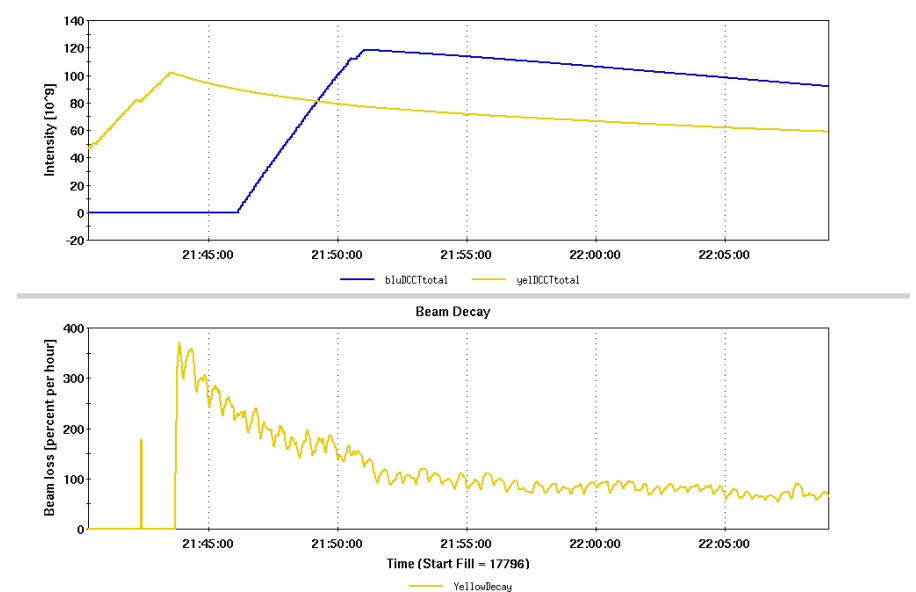

Figure 4: Beam intensities and hourly Yellow beam decay rate during injection of the Blue beam, at the FY2014 near-integer working point $\left(Q_{x}, Q_{y}\right)=(28.095,29.085)$ (bottom).

observed at these tunes, as depicted in Figure 4. The lifetime of the already stored Yellow beam continued to improve during injection of and collision with the Blue beam. When the working point was set to the 2010 tunes of $\left(Q_{x}, Q_{y}\right)=(28.13,29,12)$ in a control experiment the lifetime of the already stored Yellow beam suffered as soon as the Blue beam was injected and collided with the Yellow beam, see Figure 5.

\section{Operation with an internal halo target}

As an alternative to collider operations at very low energies, the STAR collaboration had proposed installation of an internal halo target $2.05 \mathrm{~m}$ upstream of the interaction point in the direction 


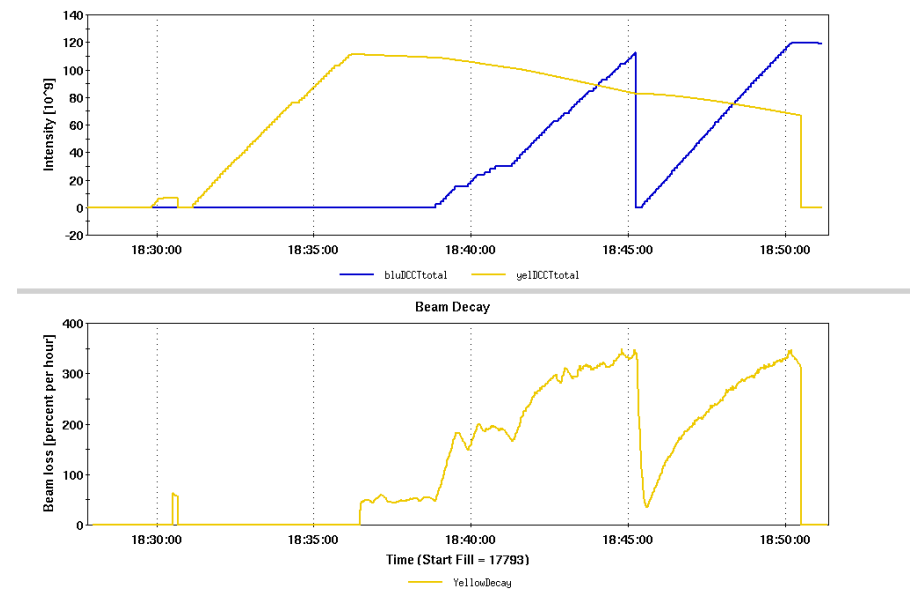

Figure 5: Beam intensities and hourly Yellow beam decay rate during injection of the Blue beam, at the FY2010 working point $\left(Q_{x}, Q_{y}\right)=(.13, .12)$ (bottom).

of the Yellow beam [5]. This target consists of a 30 mil $(750 \mu \mathrm{m})$ thick gold foil that intercepts the Yellow beam halo in the vertical plane. Only the lower part of the beam pipe is equipped with the target foil to avoid blockage of the beam pipe aperture in case of a mechanical failure of the target.

The aperture of the target needs to be chosen smaller than the $20 \mathrm{~mm}$ inner radius of the nearby detector beam pipe in order to make the target the local aperture limitation. On the other hand the target must not interfere with regular collider operation. These two objectives can be accomplished by selecting a target half aperture of $16 \mathrm{~mm}$, which provides sufficient aperture during regular operations, including the $\pm 5 \mathrm{~mm}$ separation bumps. By increasing the separation bumps and blowing up the beam emittance the target can then be made the limiting aperture in dedicated target mode, as shown in Figure 6.

Operating an internal halo target in a storage ring is not without challenges, as the experience with the HERA-B target has shown [6, 7]. Target interaction rates can vary widely due to beam orbit jitter and/or insufficient diffusion to replenish the halo. Variations in the transverse size of individual bunches result in only those bunches with the largest emittance contributing to the target interaction rate. Since the STAR target is not moveable, the interaction rate has to be controlled and maintained steady by manipulating machine parameters such as tunes to stabilize the interaction rates. Since the target will only be operated in a dedicated mode no special care has to be taken to preserve the emittance of the beam core while enhancing the halo, as would be the case in simultaneous operation with the collider mode.

In a dedicated beam experiment a vertical collimator was inserted into the halo of the stored proton beam at injection energy to simulate the STAR target. When the collimator reached a position $11.5 \mathrm{~mm}$ from the beam center, a stable loss rate of $40 \mathrm{~Hz} / \mathrm{bunch}$ was recorded by the local PIN diodes. Starting with a working point of $\left(Q_{x}, Q_{y}\right)=(28.696,29.691)$, collimator loss rate control was attempted by moving the fractional tunes closer to the nonlinear resonances at $2 / 3$ and $7 / 10$. The greatest effect on the loss rate was achieved when the vertical tune was lowered towards $2 / 3$, while the horizontal tune was kept constant at $Q_{x}=28.698$, as shown in Figure 7 . 


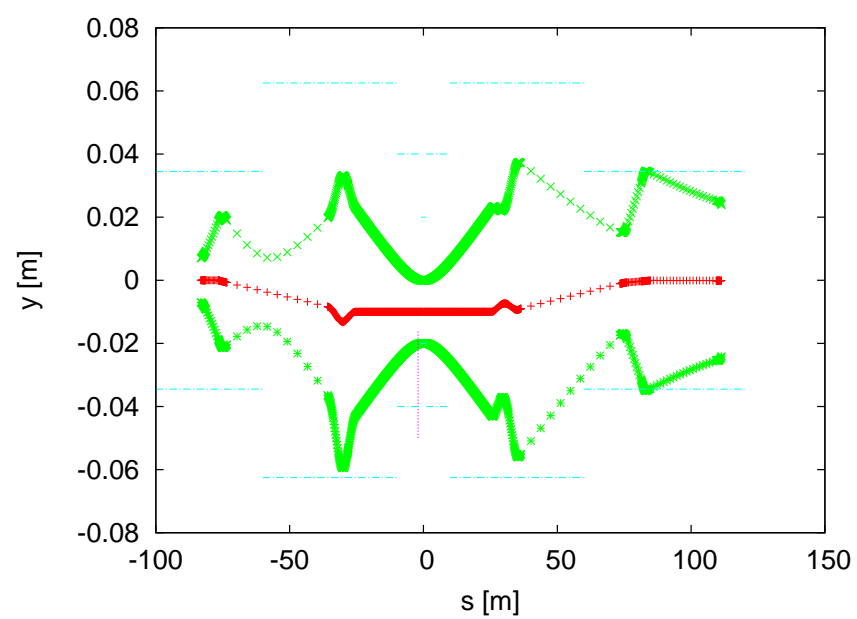

Figure 6: Geometry in target mode with $\beta^{*}=8 \mathrm{~m}$. When a $-10 \mathrm{~mm}$ orbit bump (red) is applied, the target (magenta) becomes the limiting aperture. The beam emittance is chosen such that the resulting beam envelope (green) just barely fits into the beam pipe aperture (blue).
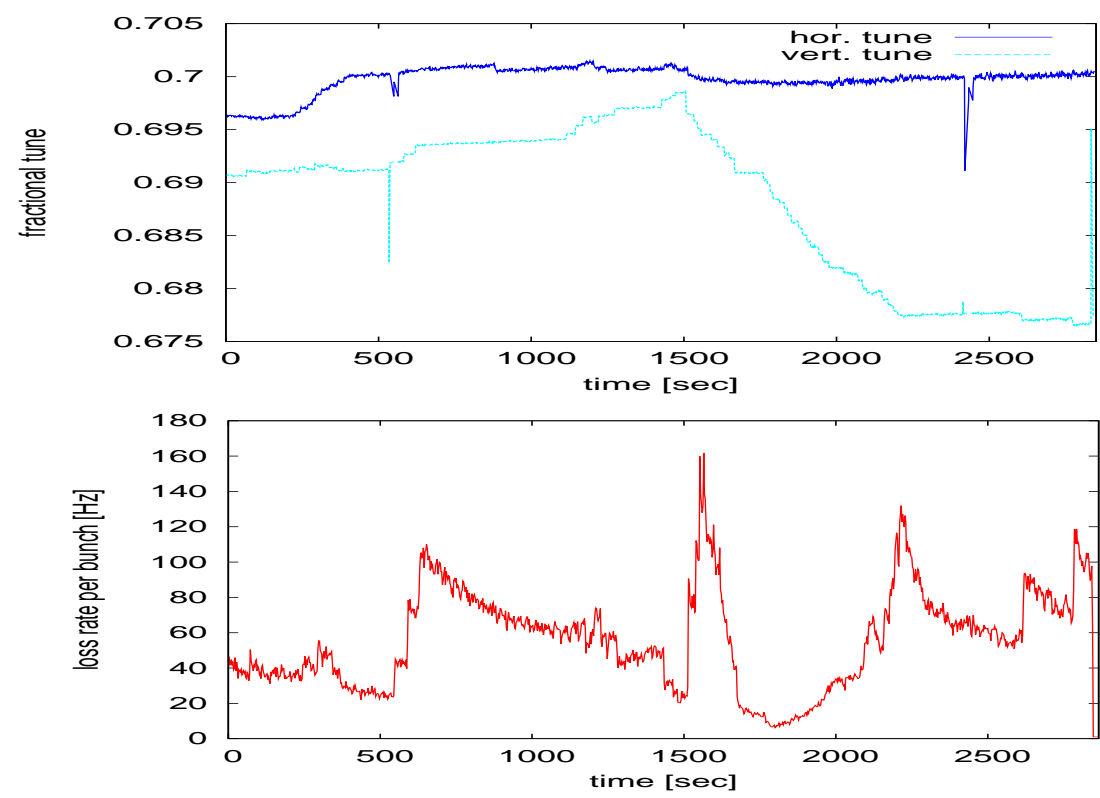

Figure 7: Loss rates during the tune scan. 

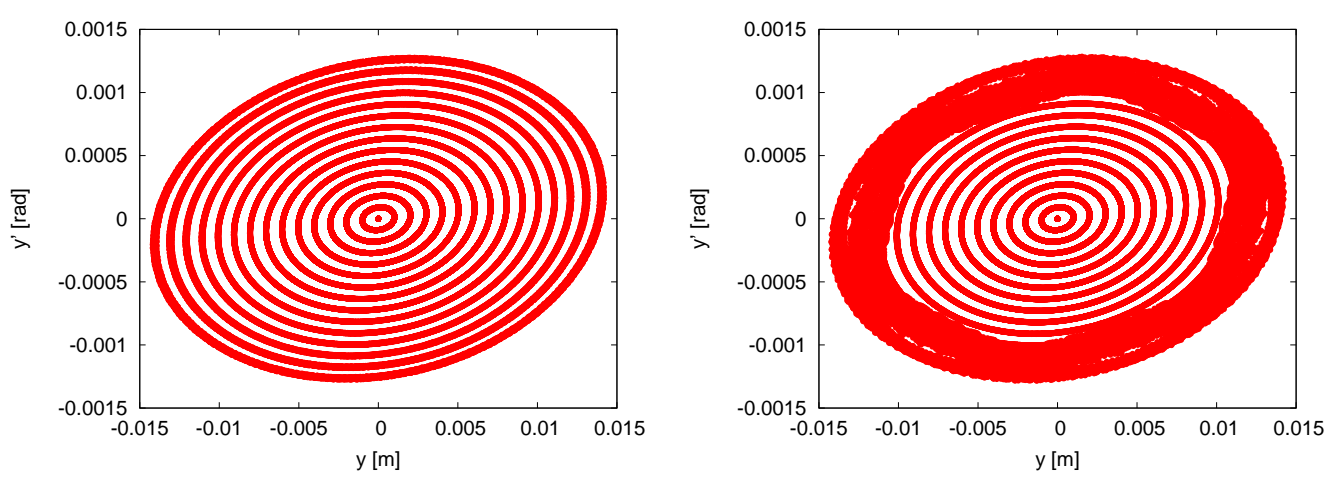

Figure 8: Vertical phase space at $\left(Q_{x}, Q_{y}\right)=(28.698,29.691)$ (left) and $(28.698,29.678)$ (right).

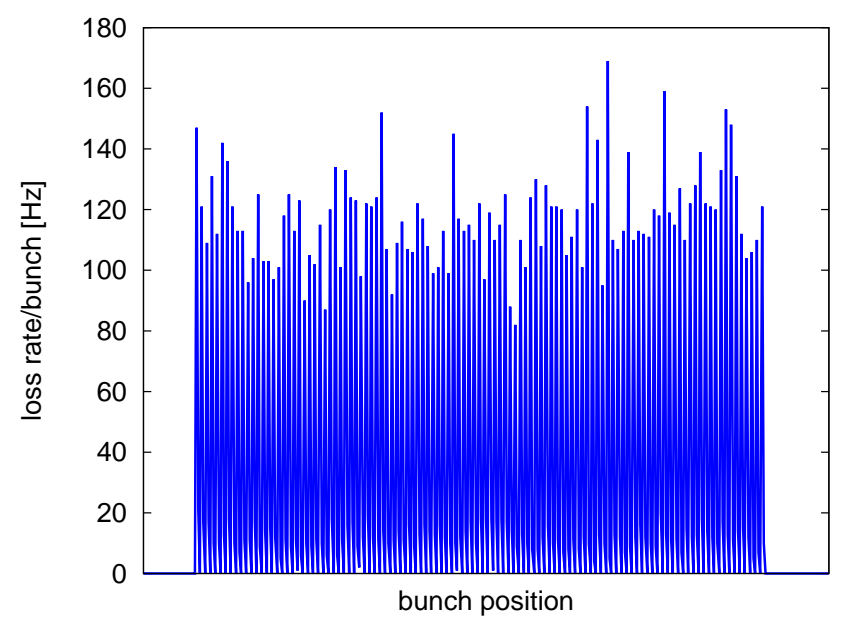

Figure 9: Bunch-by-bunch loss rates at a fixed point in time with tunes set to $\left(Q_{x}, Q_{y}\right)=(28.698,29.678)$.

This is consistent with simulation results which show regular motion in the vertical phase space for a working point of $\left(Q_{x}, Q_{y}\right)=(28.698,29.691)$, while a chaotic layer forms beginning at vertical amplitudes of $y=10 \mathrm{~mm}$ at a working point of $\left(Q_{x}, Q_{y}\right)=(28.698,29.678)$ (Fig. 8), which is in agreement with the collimator position of $11.5 \mathrm{~mm}$ during the experiment. Zooming in on the data at that latter working point shows the bunch-by-bunch structure of these collimator loss rates, as shown in Figure 9. All bunches contribute rather evenly to the overall loss rate, as would be required for smooth physics data taking at STAR. Finally, the short-term stability of the interaction rates was studied using RHIC loss monitors in the vicinity of the collimators sampled at $720 \mathrm{~Hz}$. As shown in Figure 10, loss rates fluctuate very little, indicating good stability of both the collimator position itself as well as the beam orbit.

These experiments have demonstrated the feasibility of operating RHIC with an internal target at STAR and controlling the interaction rate by adjusting the tunes. The larger intrabeam scattering rates with gold ions instead of protons are expected to improve the performance even further. 


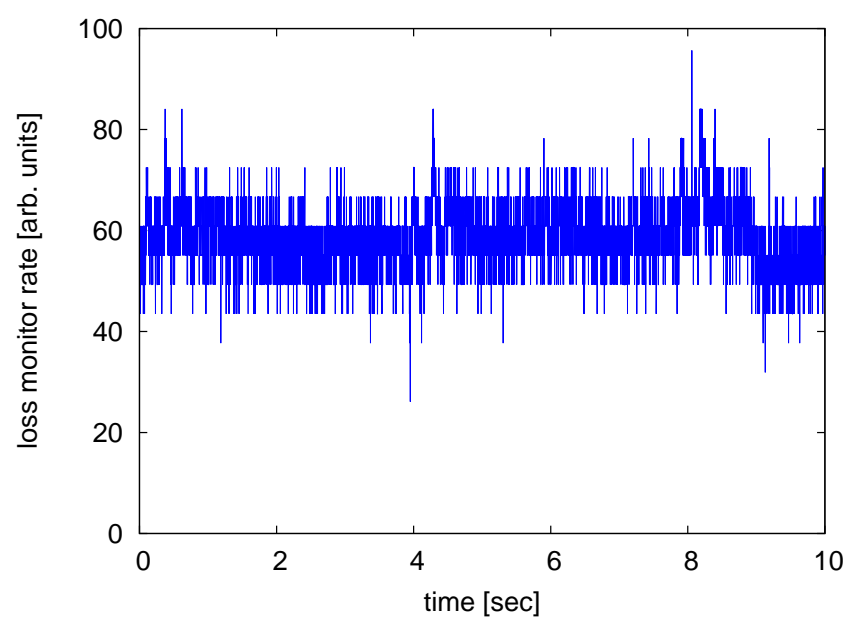

Figure 10: Loss monitor rates in the vicinity of the vertical collimator, sampled at $720 \mathrm{~Hz}$.

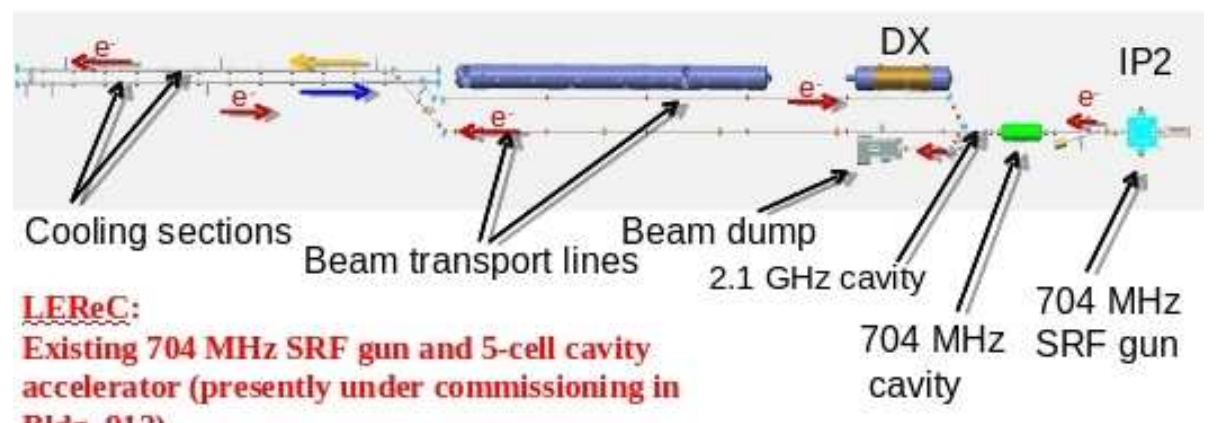

Bldg. 912)

Figure 11: Schematic drawing of the low energy RHIC electron cooler.

\section{Electron cooling}

To improve the luminosity performance at low energies for the second phase of the QCD critical point search (BES-II), an electron cooler is currently being designed. This device consists of an electron gun generating a $50 \mathrm{~mA}$ electron beam that is merged with the stored RHIC beam over a distance of $30 \mathrm{~m}$ in the 2 o'clock interaction region. Only a single electron beam will be used to cool both RHIC beams. This is accomplished by a 180 degree turn at the end of the Yellow cooling section, and then cooling the Blue beam before returning to the beam dump, as schematically depicted in Figure 11. In the first phase of the project, electrons will be accelerated to an energy between 1.6 and $2 \mathrm{MeV}$ and be discarded into a beam dump after cooling. In a second phase a 5-cell superconducting $704 \mathrm{MHz}$ cavity will be added to boost the electron beam energy to $5 \mathrm{MeV}$, thus enabling cooling of $\mathrm{Au}$ ion beams up to $10 \mathrm{GeV} /$ nucleon. This additional cavity will be run in energy-recovery mode by sending the spent electron beam after cooling back through the cavity with a 180 degree phase offset, which decelerates the beam and recovers its energy.

In contrast to all existing electron coolers which use DC beams, the low energy RHIC electron 


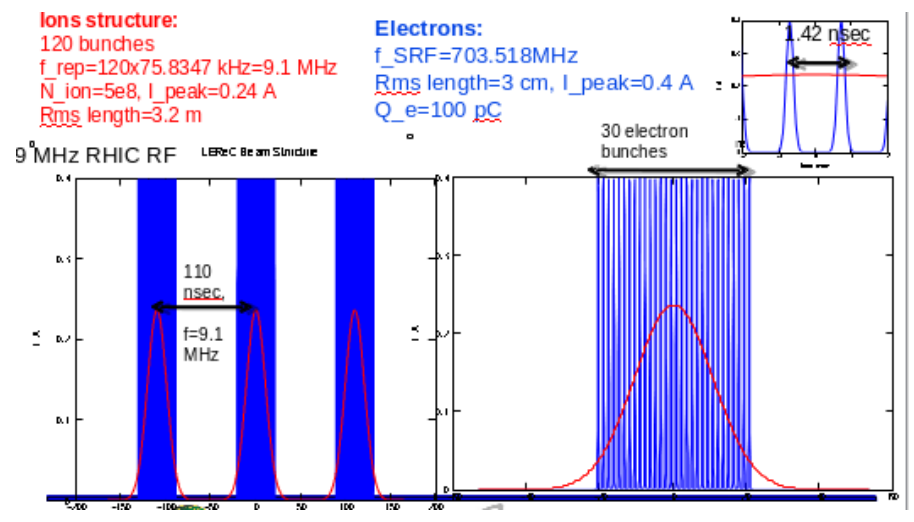

Figure 12: Electron and ion bunch structure in the low energe RHIC electron cooler.

cooler will be the first bunched electron cooler in the world. Each ion bunch is cooled by a train of 30 electron bunches $1.42 \mathrm{nsec}$ apart; the bunch trains are spaced $110 \mathrm{nsec}$ apart, corresponding to the ion bunch structure (Figure 12). A new, normal-conducting $9 \mathrm{MHz}$ RF system will be installed in RHIC to facilitate long ion bunches and therefore reduce the space charge tune shift.

This bunched electron cooler is currently being designed, and components such as the electron gun are being commissioned. Installation of the entire system is planned for FY2017, and BES-II will then take place in 2018-2019. Depending on the beam energy, luminosity enhancement factors between 4 at $3.85 \mathrm{GeV} /$ nucleon and 10 at $10 \mathrm{GeV} /$ nucleon gold ion beam energy compared to the present situation without electron cooling are expected.

\section{Summary}

RHIC performance at low energy has significantly improved based on beam experiments and simulation studies that overcame the beam lifetime reduction due to the beam-beam interaction. Electron cooling will provide an additional factor of 4 to 10 in luminosity, beginning in FY2018. First tests with of internal target operations are promising, which provides an alternative means to study heavy ion collisions at low energies.

\section{References}

[1] T. Satogata, C-A/AP/309

[2] C. Montag et al., Nucl. Instr. Meth. A564 (2006) 26-31

[3] C. Montag, "Recent Results on Beam-beam Effects in Space Charge Dominated Colliding Ion Beams at RHIC", Proc. HB2014

[4] R. Michnoff et al., MOP268, Proc. PAC 2011, 609-611

[5] STAR Beam Use Proposal 2013

[6] C. Montag et al., TPPH018, Proc. PAC 2001, 1699-1701

[7] C. Montag, AIP Conf. Proc. 693 (2004) 144-147 